\title{
The Complementary $q$-Lidstone Interpolating Polynomials and Applications
}

\author{
Zeinab Mansour ${ }^{1, *}$ and Maryam Al-Towailb ${ }^{2, *}$
}

1 Department of Mathematics, Faculty of Science, Cairo University, Giza 12613, Egypt

2 Department of Computer Science and Engineering, Faculty of Applied Studies and Community Service, King Saud University, Riyadh 11451, Saudi Arabia

* Correspondence: zeinab@sci.cu.edu.eg (Z.M.); mtowaileb@ksu.edu.sa (M.A.-T.)

Received: 30 April 2020; Accepted: 16 June 2020; Published: 19 June 2020

Abstract: In this paper, we introduce the complementary $q$-Lidstone interpolating polynomial of degree $2 n$, which involves interpolating data at the odd-order $q$-derivatives. For this polynomial, we will provide a $q$-Peano representation of the error function. Next, we use these results to prove the existence of solutions of the complementary $q$-Lidstone boundary value problems. Some examples are included.

Keywords: q-Lidstone polynomials; $q$-Peano's theorem; complementary $q$-Lidstone; boundary value problems

MSC: 05A30; 11B68; 39A05; 39A13; 30E25; 42A16

\section{Introduction}

In 1929, Lidstone [1] introduced a generalization of Taylor's series that approximates a given function in a neighborhood of two points instead of one. Recently, Ismail and Mansour [2] introduced a $q$-analog of the Lidstone expansion theorem. They proved that, under certain conditions, an entire function $f(z)$ can be expanded with respect to the points 0 and 1 in terms of the $q$-Lidstone polynomials $A_{n}(z)$ and $B_{n}(z)$ :

$$
f(z)=\sum_{n=0}^{\infty}\left[A_{n}(z) D_{q^{-1}}^{2 n} f(1)-B_{n}(z) D_{q^{-1}}^{2 n} f(0)\right] .
$$

Here, $A_{n}(z)=\eta_{q^{-1}}^{1} B_{n}(z)$ and:

$$
B_{n}(z)=\frac{2^{2 n+1}}{[2 n+1] !} B_{2 n+1}(z / 2 ; q),
$$

where $\eta_{q^{-1}}^{y} z^{n}$ denotes the $q$-translation operator defined by:

$$
\eta_{q^{-1}}^{y} z^{n}=q^{\frac{n(n-1)}{2}} z^{n}\left(-y / z ; q^{-1}\right)_{n}=y^{n}(-z / y ; q)_{n},
$$

and $B_{n}(z ; q)$ is the $q$-analogue of the Bernoulli polynomials, which is defined by the generating function:

$$
\frac{t E_{q}(z t)}{E_{q}(t / 2) e_{q}(t / 2)-1}=\sum_{n=0}^{\infty} B_{n}(z ; q) \frac{t^{n}}{[n] !},
$$


$E_{q}(z)$ and $e_{q}(z)$ are the $q$-exponential functions defined by Jackson, cf., e.g., [3,4],

$$
E_{q}(z):=\sum_{j=0}^{\infty} q^{j(j-1) / 2} \frac{z^{j}}{[j] !} ; z \in \mathbb{C} \text { and } e_{q}(z):=\sum_{j=0}^{\infty} \frac{z^{j}}{[j] !} ;|z|<1 .
$$

The $q$-Lidstone polynomials $A_{n}(z)$ and $B_{n}(z)$ of degree $(2 n+1)$ and satisfy:

$$
\begin{gathered}
A_{0}(z)=z \text { and } B_{0}(z)=z-1, \\
A_{n}(0)=A_{n}(1)=B_{n}(0)=B_{n}(1)=0, \text { for } n \in \mathbb{N}, \\
D_{q^{-1}}^{2} A_{n}(z)=A_{n-1}(z) \text { and } D_{q^{-1}}^{2} B_{n}(z)=B_{n-1}(z) .
\end{gathered}
$$

Throughout this paper, unless otherwise stated, $q$ is a positive number less than one. The sets $A_{q}$, $A_{q}^{*}$ are defined by:

$$
A_{q}:=\left\{q^{n}: n \in \mathbb{N}_{0}\right\}, \quad A_{q}^{*}:=A_{q} \cup\{0\},
$$

where $\mathbb{N}_{0}:=\{0,1,2, \ldots\}$. If $X$ is the set $A_{q}$ or $A_{q}^{*}$, then for $n>1$, we use $C_{q}^{n}(X)$ to denote the space of all continuous functions with continuous $q$-derivatives up to order $n-1$ on $X$. We shall follow the notations and terminology in $[3,5]$.

In [6], we studied the boundary value problems, which consist of an even order $q$-differential equation and the $q$-Lidstone boundary conditions. This paper extends this technique to solve the following problem:

$$
(-1)^{n} D_{q^{-1}}^{(2 n+1)} g(z)=\phi(z, g(z)), z \in A_{q}^{*}, n \in \mathbb{N},
$$

subject to the boundary conditions:

$$
g(0)=\tilde{\beta}, D_{q^{-1}}^{2 j-1} g(0)=q \tilde{\beta}_{j}, D_{q^{-1}}^{2 j-1} g(1)=q \tilde{\gamma}_{j}, \quad 1 \leq j \leq n,
$$

where $\tilde{\beta}, \tilde{\beta}_{j}, \tilde{\gamma}_{j} \in \mathbb{C}, \phi$ is a continuous real function defined on the set $A_{q}^{*} \times \mathbb{R}^{j+1}, 0 \leq j \leq 2 n$ and:

$$
g:=\left(g_{0}, g_{1}, \ldots, g_{j}\right)=\left(g, D_{q^{-1}} g, \ldots, D_{q^{-1}}^{j} g\right) \in C_{q^{-1}}^{2 n+1}\left(A_{q}^{*}\right) .
$$

We will give a $q$-analog of the complementary Lidstone interpolation, which was introduced in [7] and drawn on by Agarwal, Pinelas, and Wong in [8]. More precisely, we introduce and construct explicitly the complementary $q$-Lidstone interpolating polynomial of degree $2 n$, which involves interpolating data at the odd-order derivatives. Furthermore, we will provide a $q$-Peano representation of the error function. These results are of fundamental importance in every aspect of numerical mathematics, in the theory of $q$-differential equations such as maximum principles, $q$-boundary value problems, oscillation theory, disconjugacy, and disfocality.

This article is organized as follows. In the next section, we give the formula of the $q$-Lidstone interpolating polynomial $Q_{n}(z ; q)$ of degree $(2 n-1)$ and provide a $q$-Peano representation of the error function. In Section 3, we introduce and construct explicitly the complementary $q$-Lidstone interpolating polynomial $P_{n}(z ; q)$ of degree $2 n$, which involves interpolating data at the odd-order derivatives. In Section 4 , we are interested in the existence of solutions of the complementary $q$-Lidstone boundary value problems (3) and (4), and we will give some illustrative examples. General conclusions of this work are summarized in Section 5.

\section{Some Basic Results on the Interpolating Polynomial}

We begin by some results from [6]: 
Lemma 1. Let $g \in C^{2 n}\left(A_{q}^{*}\right)$. Then

$$
\begin{gathered}
g(x)=\sum_{m=0}^{n-1}\left[D_{q^{-1}}^{2 m} g(1) A_{m}(x)-D_{q^{-1}}^{2 m} g(0) B_{m}(x)\right] \\
+\int_{0}^{1} G_{n}(x, q t) D_{q^{-1}}^{2 n} g\left(q^{2} t\right) d_{q} t
\end{gathered}
$$

where $A_{m}$ and $B_{m}$ are $q$-Lidstone polynomials of degree $2 m+1$, and:

$$
\begin{gathered}
G(x, t)= \begin{cases}-q t(1-x), & 0 \leq t<x \leq 1 ; \\
-q x(1-t), & 0 \leq x<t \leq 1\end{cases} \\
G_{1}(x, q t):=G(x, q t) \\
G_{n}(x, q t)=\int_{0}^{1} G(x, q y) G_{n-1}(q y, q t) d_{q} y \quad(n=2,3, \ldots) \\
=\int_{0}^{1} \ldots \int_{0}^{1} G\left(x, q t_{1}\right) G\left(q t_{1}, q t_{2}\right) \ldots G\left(q t_{n-1}, q t\right) d_{q} t_{1} d_{q} t_{2} \ldots d_{q} t_{n-1} .
\end{gathered}
$$

Remark 1. For $n \in \mathbb{N}$, the function $G_{n}(z, q s)$ satisfies:

$$
0 \leq(-1)^{n} G_{n}(z, q s)=\left|G_{n}(z, q s)\right|
$$

As in the classical field of approximation theory [9], we consider the $q$-Lidstone interpolating polynomial $Q_{n}(z ; q), z \in A_{q}^{*}$, of degree $2 n-1$ satisfying the $q$-Lidstone conditions:

$$
D_{q^{-1}}^{2 j} Q_{n}(0 ; q)=\beta_{j}, D_{q^{-1}}^{2 j} Q_{n}(1 ; q)=\gamma_{j}, \quad\left(\beta_{j}, \gamma_{j} \in \mathbb{C}, j=0,1, \ldots, n-1\right)
$$

A representation of the $q$-Lidstone interpolating polynomial $Q_{n}(z ; q)$ is given by the following:

Lemma 2. The $q$-Lidstone interpolating polynomial $Q_{n}(z ; q)$ can be expressed as:

$$
Q_{n}(z ; q)=\sum_{m=0}^{n-1}\left[\gamma_{m} A_{m}(z)-\beta_{m} B_{m}(z)\right] \text {. }
$$

Proof. It is clear that $Q_{n}(z ; q)$ is a polynomial of degree at most $(2 n-1)$. From (2), we have:

$$
\begin{aligned}
\left(D_{q^{-1}}^{2 j} Q_{n}\right)(z ; q) & =\sum_{m=j}^{n-1}\left[\gamma_{m} D_{q^{-1}}^{2 j} A_{m}(z)-\beta_{m} D_{q^{-1}}^{2 j} B_{m}(z)\right] \\
& =\sum_{m=j}^{n-1}\left[\gamma_{m} A_{m-j}(z)-\beta_{m} B_{m-j}(z)\right] \\
& =\sum_{m=0}^{n-j-1}\left[\gamma_{m+j} A_{m}(z)-\beta_{m+j} B_{m}(z)\right] .
\end{aligned}
$$

It follows that:

$$
\left(D_{q^{-1}}^{2 j} Q_{n}\right)(0 ; q)=-\left.\beta_{j} B_{0}(z)\right|_{z=0}=\beta_{j}, \quad 0 \leq j \leq n-1,
$$

and:

$$
\left(D_{q^{-1}}^{2 j} Q_{n}\right)(1 ; q)=\left.\gamma_{j} A_{0}(z)\right|_{z=1}=\gamma_{j}, \quad 0 \leq j \leq n-1
$$


Let $\left(D_{q^{-1}}^{2 j} f\right)(0)=\beta_{j}$ and $\left(D_{q^{-1}}^{2 j} f\right)(1)=\gamma_{j}(0 \leq j \leq n-1)$ where $f \in C^{2 n}\left(A_{q}^{*}\right)$. In such a case, $Q_{n}(z ; q)$ is called the $q$-Lidstone interpolating polynomial of the function $f(z)$. For the associated error:

$$
R(z ; q)=f(z)-Q_{n}(z ; q)
$$

we provide a $q$-Peano representation. Therefore, in the following, we recall a $q$-Peano kernel theorem from [10], which is an important role in our results.

We use the notation $\mathcal{P}_{n}$ to denote the space of polynomials of degree $n$, and we consider functions of class $C_{q}^{n+1}\left(A_{q}^{*}\right)$.

Define the two variables polynomials $\phi_{n}(z, t), z, t \in \mathbb{C}$, to be:

$$
\phi_{0}(z, t):=1, \quad \phi_{n}(z, t):= \begin{cases}z^{n}\left(\frac{t}{z} ; q\right)_{n}, & z \neq 0 \\ (-1)^{n} q^{\frac{n(n-1)}{2}} t^{n}, & z=0 .\end{cases}
$$

Theorem 1. ( $q$-Peano kernel theorem) Let $L$ be a linear functional defined over $C_{q}^{n+1}\left(A_{q}^{*}\right)$. If $L(p)=0$ for all polynomials $p(z)$ of degree $n$, then for all $f \in C_{q}^{n+1}\left(A_{q}^{*}\right)$ :

$$
L(f)=\int_{0}^{1}\left(D_{q}^{n+1} f\right)(t) K_{n}(z, q t) d_{q} t
$$

where:

$$
K_{n}(z, q t)=\frac{1}{[n]_{q} !} L_{z}\left(\phi_{n}^{+}(z, q t)\right)
$$

here, $L_{z}$ means the linear functional L applied to $\phi_{n}^{+}(z, q t)$ as a function of $z$, and:

$$
\phi_{n}^{+}(z, q t):= \begin{cases}\phi_{n}(z, q t), & z \geq t \\ 0, & z<t .\end{cases}
$$

Let $z_{0}, z_{1}, \ldots, z_{n}$ be distinct points in $A_{q}^{*}$. We denote by $I_{k}(z), k=0,1, \ldots n$, to the polynomials that are defined on $A_{q}^{*}$ and satisfy the following condition:

$$
\sum_{k=0}^{n} I_{k}(z)=1, \quad \sum_{k=0}^{n} r\left(z_{k}\right) I_{k}(z)=r(z), \text { for } r \in \mathcal{P}_{n} .
$$

Lemma 3. (see [10]) Suppose $z_{0}, z_{1}, \ldots, z_{n}$ are distinct points in $A_{q}^{*}$. Define the corresponding error functional by:

$$
L_{z}(f)=f(z)-\sum_{k=0}^{n} f\left(z_{k}\right) I_{k}(z)
$$

Then:

$$
[n]_{q} ! L(f)=\sum_{k=0}^{n} I_{k}(z) \int_{t=z_{k}}^{z} \phi_{n}\left(z_{k}, t\right)\left(D_{q}^{n+1} f\right)(t) d_{q} t
$$

Now, we prove the main result.

Theorem 2. Let $f \in C^{2 n}\left(A_{q}^{*}\right)$. Then:

$$
R(z ; q)=\int_{0}^{1} G_{n}(z, q t)\left(D_{q^{-1}}^{2 n} f\right)\left(q^{2} t\right) d_{q} t
$$


here, $G_{n}$ has a $q$-Peano representation:

$$
G_{n}(z, q t)=- \begin{cases}\sum_{i=0}^{n-1} \frac{q^{2(n-i)^{2}+3 i-n}}{[2 n-2 i-1] q !} A_{i}(z)\left(q^{1+2 i} t ; q\right)_{2 n-2 i-1}, & z<t \\ \sum_{i=0}^{n-1} \frac{q^{2(2 n-1)(n-i)}}{[2 n-2 i-1] q !} B_{i}(z)(-t)^{2 n-2 i-1}, & z \geq t .\end{cases}
$$

Proof. According to Lemma 2, the $q$-Lidstone interpolating polynomial of the function $f$ can be expressed as:

$$
Q_{n}(z ; q)=\sum_{i=0}^{n-1}\left[\left(D_{q^{-1}}^{2 i} f\right)(1) A_{i}(z)-\left(D_{q^{-1}}^{2 i} f\right)(0) B_{i}(z)\right],
$$

where the associated error:

$$
\begin{aligned}
R(z ; q) & =f(z)-Q_{n}(z ; q) \\
& =f(z)-\sum_{i=0}^{n-1}\left[\left(D_{q^{-1}}^{2 i} f\right)(1) A_{i}(z)-\left(D_{q^{-1}}^{2 i} f\right)(0) B_{i}(z)\right] .
\end{aligned}
$$

Therefore, from Lemma 1, we obtain (11).

Now, we apply Theorem 1 . Note that, the reminder $L(f)$ defined by:

$$
L(f):=R(z ; q)=\int_{0}^{1} G_{n}(z, q t)\left(D_{q^{-1}}^{2 n} f\right)\left(q^{2 n} t\right) d_{q} t,
$$

where:

$$
G_{n}(z, q t):=K_{2 n-1}(z, q t)=\frac{q^{n(2 n-1)}}{[2 n-1]_{q} !} L_{z}\left(\phi_{2 n-1}^{+}(z, q t)\right) .
$$

By Equation (12), we obtain:

$$
\begin{gathered}
L_{z}\left(\phi_{2 n-1}^{+}(z, q t)\right)= \\
\phi_{2 n-1}^{+}(z, q t)-\sum_{i=0}^{n-1}\left[\left(D_{q^{-1}}^{2 i} \phi_{2 n-1}^{+}\right)(1) A_{i}(z)-\left(D_{q^{-1}}^{2 i} \phi_{2 n-1}^{+}\right)(0) B_{i}(z)\right] .
\end{gathered}
$$

We can verify that:

$$
\begin{aligned}
& \left(D_{q^{-1}}^{2 i} \phi_{2 n-1}\right)(z, q t)=\frac{[2 n-1]_{q} !}{[2 n-2 i-1]_{q} !} q^{i-2 i^{2}} \phi_{2 n-2 i-1}\left(q^{-2 i} z, q t\right) \\
& = \begin{cases}\frac{[2 n-1]_{q} !}{[2 n-2 i-1]_{q} !} q^{i-2 i^{2}}\left(z q^{-2 i}\right)^{2 n-2 i-1}\left(q^{1+2 i} t / z ; q\right)_{2 n-2 i-1}, & z \neq 0 \\
\frac{[2 n-1]_{q} !}{[2 n-2 i-1]_{q} !} q^{i-2 i^{2}+(2 n-2 i-1)(n-i)}(-t)^{2 n-2 i-1}, & z=0 .\end{cases}
\end{aligned}
$$

Therefore, by Lemma 3, we conclude that $G_{n}$ has a $q$-Peano representation:

$$
G_{n}(z, q t)=- \begin{cases}\sum_{i=0}^{n-1} \frac{\left.q^{2(n-i)}\right)^{2}+3 i-n}{[2 n-2 i-1] q !} A_{i}(z)\left(q^{1+2 i} t ; q\right)_{2 n-2 i-1}, & \mathrm{z}<\mathrm{t} \\ \sum_{i=0}^{n-1} \frac{q^{2(2 n-1)(n-i)}}{[2 n-2 i-1]_{q} !} B_{i}(z)(-t)^{2 n-2 i-1,} & \mathrm{z} \geq \mathrm{t} .\end{cases}
$$




\section{The Complementary $q$-Lidstone Interpolating Polynomials}

In this section, we consider the complementary $q$-Lidstone interpolating polynomial $P_{n}(z ; q)$ in $A_{q}^{*}$, which is of degree $2 n$ and satisfies the conditions:

$$
P_{n}(0 ; q)=\tilde{\beta}, \quad\left(D_{q^{-1}}^{2 i-1} P_{n}\right)(0 ; q)=\tilde{\beta}_{i}, \quad\left(D_{q^{-1}}^{2 i-1} P_{n}\right)(1 ; q)=\tilde{\gamma}_{i}
$$

where $\tilde{\beta}, \tilde{\beta}_{i}, \tilde{\gamma}_{i} \in \mathbb{C}, 1 \leq i \leq m$.

In the next result, we denote by $v_{m}(z)$ and $\tau_{m}(z)(m \geq 0)$ the first $q^{-1}$-derivatives of $A_{m}(z)$ and $B_{m}(z)$, respectively. That is,

$$
D_{q^{-1}} A_{m}(z)=v_{m}(z) \text { and } D_{q^{-1}} B_{m}(z)=\tau_{m}(z), m \geq 0 .
$$

Then, it immediately follows that:

1. $v_{0}(z)=1=\tau_{0}(z)$;

2. $D_{q^{-1}} v_{m}(z)=A_{m-1}(z)$;

3. $D_{q^{-1}} \tau_{m}(z)=B_{m-1}(z)$;

4. $\quad \int_{0}^{q} v_{m}(s) d_{q} s=\int_{0}^{q} \tau_{m}(s) d_{q} s=0, m \geq 1$;

5. $\quad \int_{0}^{z} \tau_{m-1}(s) d_{q} s=D_{q^{-1}} \tau_{m}(z), m \geq 1$.

Theorem 3. Let $g \in C^{2 n+1}\left(A_{q}^{*}\right)$ and $P_{n}(z ; q)$ be the complementary $q$-Lidstone interpolating polynomial of degree $2 n$ of the function $g(z)$. Then:

$$
g(z)=P_{n}(z ; q)+\widetilde{R}(z ; q)
$$

where:

$$
\begin{aligned}
P_{n}(z ; q)= & g(0)+\frac{1}{q} \sum_{m=1}^{n}\left[\left(D_{q^{-1}}^{2 m-1} g\right)(1)\left(v_{m}(z)-v_{m}(0)\right)\right]+ \\
& \frac{1}{q} \sum_{m=1}^{n}\left[\left(D_{q^{-1}}^{2 m-1} g\right)(0)\left(\tau_{m}(0)-\tau_{m}(z)\right)\right],
\end{aligned}
$$

and $\widetilde{R}(z ; q)$ is the residue term:

$$
\widetilde{R}(z ; q)=\int_{0}^{1} H_{n}(z, q s)\left(D_{q^{-1}}^{2 n+1} g\right)\left(q^{2} s\right) d_{q} s .
$$

Furthermore, the kernel $H_{n}(z, q s)$ has the $q$-Peano representation:

$$
\begin{gathered}
H_{n}(z, q s)=\int_{0}^{q z} G_{n}(t, q s) d_{q} t \\
=\frac{1}{q} \sum_{m=1}^{n} \frac{q^{2(n-m+1)^{2}+3(m-1)-n}}{[2 n-2 m+1]_{q} !}\left(v_{m}(0)-v_{m}(z)\right)\left(q^{2 m-1} s ; q\right)_{2 n-2 m+1} ; z<s,
\end{gathered}
$$

and for $z \geq s$,

$$
H_{n}(z, q s)=\frac{q^{4 n^{2}-1} s^{2 n}}{[2 n]_{q} !}+\frac{1}{q} \sum_{m=1}^{n} \frac{q^{2(2 n-1)(n-m+1)}}{[2 n-2 m+1]_{q} !}\left(\tau_{m}(z)-\tau_{m}(0)\right) s^{2 n-2 m+1} .
$$


Proof. Let $f=D_{q^{-1}} g$. Integrate both sides of (7) from zero to $q z$, to obtain:

$$
\begin{aligned}
& \int_{0}^{q z} D_{q^{-1}} g(t) d_{q} t=g(z)-g(0) \\
& =\sum_{m=0}^{n-1}\left[\left(D_{q^{-1}}^{2 m+1} g\right)(1) \int_{0}^{q z} A_{m}(t) d_{q} t-\left(D_{q^{-1}}^{2 m+1} g\right)(0) \int_{0}^{q z} B_{m}(t) d_{q} t\right] \\
& +\int_{0}^{q z}\left(\int_{0}^{1} G_{n}(t, q s)\left(D_{q^{-1}}^{2 n+1} g\right)\left(q^{2} s\right) d_{q} s\right) d_{q} t .
\end{aligned}
$$

From (2), we have:

$$
\begin{aligned}
\int_{0}^{q z} A_{m}(t) d_{q} t & =\int_{0}^{q z} D_{q^{-1}}^{2} A_{m+1}(t) d_{q} t \\
& =\int_{0}^{q z} \frac{1}{q} D_{q}^{2} A_{m+1}\left(t / q^{2}\right) d_{q} t \\
& =\frac{1}{q}\left[D_{q^{-1}} A_{m+1}(z)-D_{q^{-1}} A_{m+1}(0)\right] \\
& =\frac{1}{q}\left[v_{m+1}(z)-v_{m+1}(0)\right], \quad m \in \mathbb{N}_{0} .
\end{aligned}
$$

Similarly, we can verify that:

$$
\int_{0}^{q z} B_{m}(t) d_{q} t=\frac{1}{q}\left[\tau_{m+1}(z)-\tau_{m+1}(0)\right]
$$

It follows

$$
\begin{aligned}
& g(z)=\int_{0}^{q z}\left(\int_{0}^{1} G_{n}(t, q s)\left(D_{q^{-1}}^{2 n+1} g\right)\left(q^{2} s\right) d_{q} s\right) d_{q} t+g(0) \\
& +\frac{1}{q} \sum_{m=1}^{n}\left[\left(D_{q^{-1}}^{2 m-1} g\right)(1)\left(v_{m}(z)-v_{m}(0)\right)+\left(D_{q^{-1}}^{2 m-1} g\right)(0)\left(\tau_{m}(0)-\tau_{m}(z)\right)\right]
\end{aligned}
$$

and then, we get Equation (13), where:

$$
\begin{aligned}
P_{n}(z ; q)= & g(0)+\frac{1}{q} \sum_{m=1}^{n}\left[\left(D_{q^{-1}}^{2 m-1} g\right)(1)\left(v_{m}(z)-v_{m}(0)\right)+\right. \\
& \left.\left(D_{q^{-1}}^{2 m-1} g\right)(0)\left(\tau_{m}(0)-\tau_{m}(z)\right)\right] \\
\widetilde{R}(z ; q)= & \int_{0}^{1}\left(\int_{0}^{q z} G_{n}(t, q s) d_{q} t\right)\left(D_{q^{-1}}^{2 n+1} g\right)\left(q^{2} s\right) d_{q} s \\
= & \int_{0}^{1} H_{n}(z, q s)\left(D_{q^{-1}}^{2 n+1} g\right)\left(q^{2} s\right) d_{q} s .
\end{aligned}
$$

By using Theorem 2, for $z<s$, we obtain:

$$
\begin{aligned}
& H_{n}(z, q s)=\int_{0}^{q z} G_{n}(t, q s) d_{q} t \\
& =-\sum_{m=0}^{n-1} \frac{q^{2(n-m)^{2}+3 m-n}}{[2 n-2 m-1]_{q} !}\left(\int_{0}^{q z} A_{m}(t) d_{q} t\right)\left(q^{1+2 m} s ; q\right)_{2 n-2 m-1} \\
& =-\frac{1}{q} \sum_{m=1}^{n} \frac{q^{2(n-m+1)^{2}+3(m-1)-n}}{[2 n-2 m+1]_{q} !}\left(v_{m}(z)-v_{m}(0)\right)\left(q^{2 m-1} s ; q\right)_{2 n-2 m+1} .
\end{aligned}
$$


Similarly, for $z \geq s$, we have:

$$
\begin{aligned}
& H_{n}(z, q s)=\int_{0}^{q z} G_{n}(t, q s) d_{q} t=\int_{0}^{q s} G_{n}(t, q s) d_{q} t+\int_{q s}^{q z} G_{n}(t, q s) d_{q} t \\
& =-\frac{1}{q} \sum_{m=1}^{n} \frac{q^{2(n-m+1)^{2}+3(m-1)-n}}{[2 n-2 m+1]_{q} !}\left(v_{m}(s)-v_{m}(0)\right)\left(q^{2 m-1} s ; q\right)_{2 n-2 m+1} \\
& +\frac{1}{q} \sum_{m=1}^{n} \frac{q^{2(2 n-1)(n-m+1)}}{[2 n-2 m+1]_{q} !}\left(\tau_{m}(z)-\tau_{m}(s)\right)(s)^{2 n-2 m+1} .
\end{aligned}
$$

Finally, we will take $g(z)=\frac{q^{2 n^{2}+n-1}}{[2 n] q !} \phi_{2 n}(z, t)$, where $\phi_{2 n}(z, t)$ is the polynomial function of degree $2 n$ defined in (8). Then, after some calculations, we verify that:

$$
D_{q^{-1}, z}^{2 m-1} g(z)=\frac{q^{2 n^{2}+n-1}}{[2 n-2 m+1]_{q} !} q^{(m-1)(1-2 m)} \phi_{2 n-2 m+1}\left(z q^{1-2 m}, s\right)
$$

Hence, we obtain:

$$
\begin{aligned}
\left(D_{q^{-1}}^{2 m-1} g\right)(1) & =\frac{q^{2(n-m+1)^{2}+3(m-1)-n}}{[2 n-2 m+1]_{q} !}\left(q^{2 m-1} s ; q\right)_{2 n-2 m+1}, \\
\left(D_{q^{-1}}^{2 m-1} g\right)(0) & =\frac{q^{2(2 n-1)(n-m+1)}}{[2 n-2 m+1]_{q} !}(-s)^{2 n-2 m+1}, \\
g(0) & =\frac{q^{4 n^{2}-1}}{[2 n]_{q} !} s^{2 n} .
\end{aligned}
$$

By using (14), we get:

$$
\begin{gathered}
\frac{q^{2 n^{2}+n-1}}{[2 n]_{q} !} \phi_{2 n}(z, t)= \\
\frac{q^{4 n^{2}-1}}{[2 n]_{q} !} s^{2 n}+\frac{1}{q} \sum_{m=1}^{n} \frac{q^{2(2 n-1)(n-m+1)}}{[2 n-2 m+1]_{q} !}\left(\tau_{m}(0)-\tau_{m}(z)\right)(-s)^{2 n-2 m+1} \\
+\frac{1}{q} \sum_{m=1}^{n} \frac{q^{2(n-m+1)^{2}+3(m-1)-n}}{[2 n-2 m+1]_{q} !}\left(v_{m}(z)-v_{m}(0)\right)\left(q^{2 m-1} s ; q\right)_{2 n-2 m+1} .
\end{gathered}
$$

Therefore, for $z=s$, we have:

$$
\begin{aligned}
& \frac{1}{q} \sum_{m=1}^{n} \frac{q^{2(n-m+1)^{2}+3(m-1)-n}}{[2 n-2 m+1]_{q} !}\left(v_{m}(s)-v_{m}(0)\right)\left(q^{2 m-1} s ; q\right)_{2 n-2 m+1}= \\
& \frac{1}{q} \sum_{m=1}^{n} \frac{q^{2(2 n-1)(n-m+1)}}{[2 n-2 m+1]_{q} !}\left(\tau_{m}(0)-\tau_{m}(s)\right)(s)^{2 n-2 m+1}-\frac{q^{4 n^{2}-1}}{[2 n]_{q} !} s^{2 n} .
\end{aligned}
$$

Combining (17) and (18), for $z \geq s$, we get:

$$
H_{n}(z, q s)=\frac{q^{4 n^{2}-1} s^{2 n}}{[2 n]_{q} !}+\frac{1}{q} \sum_{m=1}^{n} \frac{q^{2(2 n-1)(n-m+1)}}{[2 n-2 m+1]_{q} !}\left(\tau_{m}(z)-\tau_{m}(0)\right) s^{2 n-2 m+1} .
$$

This completes the proof. 
Remark 2. by using Remark 1, we obtain:

$$
0 \leq(-1)^{n} H_{n}(z, q s)=\left|H_{n}(z, q s)\right|
$$

Lemma 4. (see [6]) For $z \in A_{q}^{*}$, there exist some constants $C_{n}$, such that:

$$
\begin{gathered}
\int_{0}^{1}\left|G_{n}\left(z, q^{n} s\right)\right| d_{q} t \leq \frac{(1-q)^{2 n}}{q^{n(n-3 / 2)}} C_{n} \\
\int_{0}^{1}\left|D_{q^{-1}, z} G_{n}\left(z, q^{n} s\right)\right| d_{q} t \leq \frac{(1-q)^{2(n-1)}}{q^{(n-1)(n-5 / 2)}}\left(q+\frac{q^{2}}{1+q}\right) C_{n} .
\end{gathered}
$$

Lemma 5. There exist some constants $C_{2 n+1, k}$ such that:

$$
\left|\left(D_{q^{-1}}^{k} g\right)(z)-\left(D_{q^{-1}}^{k} P_{n}\right)(z ; q)\right| \leq C_{2 n+1, k} \max _{z \in A_{q}^{*}}\left|\left(D_{q^{-1}}^{2 n+1} g\right)\left(q^{2} z\right)\right|,
$$

for $k=0,1, \ldots, 2 n$.

Proof. From (13) and (15), we get:

$$
\begin{aligned}
\left|g(z)-P_{n}(z ; q)\right| & =\left|\int_{0}^{1} H_{n}(z, q s)\left(D_{q^{-1}}^{2 n+1} g\right)\left(q^{2} s\right) d_{q} s\right| \\
& \leq \max _{z \in A_{q}^{*}}\left(\int_{0}^{1}\left|H_{n}(z, q s)\right| d_{q} s\right) \max _{z \in A_{q}^{*}}\left|\left(D_{q^{-1}}^{2 n+1} g\right)\left(q^{2} z\right)\right| .
\end{aligned}
$$

Note that:

$$
\int_{0}^{1}\left|H_{n}(z, q s)\right| d_{q} s \leq \int_{0}^{1} \int_{0}^{q z}\left|G_{n}(t, q s)\right| d_{q} t d_{q} s,
$$

and from Lemma 4, we conclude that the double $q$-integral on the right-hand side of (22) is absolutely convergent. Therefore, we can interchange the order of the $q$-integrations to obtain:

$$
\begin{aligned}
\int_{0}^{1}\left|H_{n}(z, q s)\right| d_{q} s & \leq \int_{0}^{q z}\left(\int_{0}^{1}\left|G_{n}(t, q s)\right| d_{q} s\right) d_{q} t \\
& \leq \frac{q(1-q)^{2 n}}{q^{n\left(n-\frac{3}{2}\right)}} C_{n} z
\end{aligned}
$$

Since $z \in A_{q}^{*}$, we obtain:

$$
\int_{0}^{1}\left|H_{n}(z, q s)\right| d_{q} s \leq C_{2 n+1,0}
$$

where $C_{2 n+1,0}=\frac{q(1-q)^{2 n}}{q^{n\left(n-\frac{3}{2}\right)}} C_{n}$. Combining (21) with (23), we get the result for $k=0$.

Again, by using (13) and (15), we get:

$$
D_{q^{-1}}^{(k)} g(z)-D_{q^{-1}}^{(k)} P_{n}(z ; q)=\int_{0}^{1} D_{q^{-1}}^{k-1} G_{n}(z, q s)\left(D_{q^{-1}}^{(2 n+1)} g\right)\left(q^{2} s\right) d_{q} s .
$$


Hence, using (6), (16), and Lemma 4, we obtain:

$$
\begin{aligned}
& \left|D_{q^{-1}}^{2 k-1} g(z)-D_{q^{-1}}^{2 k-1} P_{n}(z ; q)\right| \\
& \leq \max _{z \in A_{q}^{*}}\left(\int_{0}^{1}\left|D_{q^{-1}}^{2 k-2} G_{n}(z, q s)\right| d_{q} s\right) \max _{z \in A_{q}^{*}}\left|\left(D_{q^{-1}}^{2 n+1} g\right)\left(q^{2} z\right)\right| \\
& =\max _{z \in A_{q}^{*}}\left(\int_{0}^{1}\left|G_{n-k+1}(z, q s)\right| d_{q} s\right) \max _{z \in A_{q}^{*}}\left|\left(D_{q^{-1}}^{2 n+1} g\right)\left(q^{2} z\right)\right| \\
& \leq \frac{(1-q)^{2(n-k+1)}}{q^{(n-k+1)\left(n-k-\frac{1}{2}\right)}} C_{n} \max _{z \in A_{q}^{*}}\left|\left(D_{q^{-1}}^{2 n+1} g\right)\left(q^{2} z\right)\right| \\
& =C_{2 n+1,2 k-1} \max _{z \in A_{q}^{*}}\left|\left(D_{q^{-1}}^{2 n+1} g\right)\left(q^{2} z\right)\right|, \quad 1 \leq k \leq n .
\end{aligned}
$$

Similarly, we conclude that:

$$
\begin{aligned}
& \left|D_{q^{-1}}^{2 k} g(z)-D_{q^{-1}}^{2 k} P_{n}(z ; q)\right| \\
& \leq \max _{z \in A_{q}^{*}}\left(\int_{0}^{1}\left|D_{q^{-1}}^{2 k-1} G_{n}(z, q s)\right| d_{q} s\right) \max _{z \in A_{q}^{*}}\left|\left(D_{q^{-1}}^{2 n+1} g\right)\left(q^{2} z\right)\right| \\
& \leq \frac{(1-q)^{2(n-k)}}{q^{(n-k)\left(n-k-\frac{3}{2}\right)}}\left(q+\frac{q^{2}}{1+q}\right) C_{n} \max _{z \in A_{q}^{*}}\left|\left(D_{q^{-1}}^{2} g\right)\left(q^{2 n+1} z\right)\right| \\
& =C_{2 n+1,2 k} \max _{z \in A_{q}^{*}}\left|\left(D_{q^{-1}}^{2 n+1} g\right)\left(q^{2} z\right)\right|, \quad 1 \leq k \leq n .
\end{aligned}
$$

This completes the proof.

\section{Applications}

In this section, we present the necessary and sufficient conditions for the existence of solutions of the complementary $q$-Lidstone boundary value problem (3) and (4).

The proof depends on the results obtained in Section 3 and the Arzelà-Ascoli theorem [11].

Theorem 4. Suppose that $Q_{k}>0,0 \leq k \leq j$ are given real numbers, and define the nonzero constant $M$ to be the maximum of $\left|\phi\left(z, g_{0}, g_{1}, \ldots, g_{j}\right)\right|$ on the set $A_{q}^{*} \times E$, where:

$$
E=\left\{\left(g_{0}, g_{1}, \ldots, g_{j}\right),\left|g_{k}\right| \leq 2 Q_{k}, 0 \leq k \leq j\right\} .
$$

Furthermore, suppose that:

$$
M C_{2 n+1, k} \leq Q_{k}, \quad \max _{z \in A_{q}^{*}}\left|D_{q^{-1}}^{(k)} P_{n}(z ; q)\right|=\tilde{p_{k}} \leq Q_{k} .
$$

Then, the boundary value problem (3) and (4) has a solution in E.

Proof. First, we define the set:

$$
J\left(A_{q}^{*}\right):=\left\{g \in C_{q^{-1}}^{j}\left(A_{q}^{*}\right):\left\|D_{q^{-1}}^{k} g\right\|=\max _{z \in A_{q}^{*}}\left|D_{q^{-1}}^{j} g\right| \leq 2 Q_{k}, 0 \leq k \leq j\right\} .
$$


Notice, we can verify that $J\left(A_{q}^{*}\right)$ is a closed convex subset of the space $C_{q^{-1}}^{j}\left(A_{q}^{*}\right)$. Consider an operator $T: C_{q^{-1}}^{j}\left(A_{q}^{*}\right) \rightarrow C_{q^{-1}}^{2 n}\left(A_{q}^{*}\right)$ as follows:

$$
(T g)(z)=P_{n}(z ; q)+\int_{0}^{1}\left|H_{n}(z, q s)\right| \phi(s, g(s)) d_{q} s .
$$

In view of Theorem 3, any fixed point of (25) is a solution of the complementary boundary value problem (3) and (4).

Next, we prove that $T$ maps $J\left(A_{q}^{*}\right)$ into itself. Let $g(z) \in J\left(A_{q}^{*}\right)$. Then, from (24), (25), and Lemma 5, we get:

$$
\begin{aligned}
\left|D_{q^{-1}}^{(k)}(T g)(z)\right| & \leq\left|D_{q^{-1}}^{(k)} P_{n}(z ; q)\right|+M \int_{0}^{1}\left|D_{q^{-1}}^{(k)} H_{n}(z, q s)\right| d_{q} s \\
& \leq Q_{k}+M C_{2 n+1, k} \leq 2 Q_{k}, \quad 0 \leq k \leq j .
\end{aligned}
$$

Thus, $T\left(J\left(A_{q}^{*}\right)\right) \subseteq J\left(A_{q}^{*}\right)$. Furthermore, since $J\left(A_{q}^{*}\right)$ is a compact set, Inequality (26) implies that the sets:

$$
\mathcal{F}_{k}:=\left\{D_{q^{-1}}^{(k)}(T g)(z): g(z) \in J\left(A_{q}^{*}\right), \quad 0 \leq k \leq j\right\},
$$

are bounded and then uniformly equi-continuous on $J\left(A_{q}^{*}\right)$. Therefore, from the Arzelà-Ascoli theorem, the closure of $T\left(J\left(A_{q}^{*}\right)\right)$ is compact. Thus, by the Schauder fixed point theorem, we can find a fixed point of $T$ in $E$ that satisfies the boundary value problem (3) and (4).

Corollary 1. Assume that the function $\phi\left(z, g_{0}, g_{1}, \ldots, g_{j}\right)$ satisfies the following condition on $A_{q}^{*} \times \mathbb{R}^{j+1}$ :

$$
\left|\phi\left(z, g_{0}, g_{1}, \ldots, g_{j}\right)\right| \leq L+\sum_{k=0}^{j} L_{k}\left|g_{k}\right|^{\alpha_{k}},
$$

where $L, L_{k}$ are nonnegative constants and $0 \leq \alpha_{k}<1$. Then, the boundary value problem (3) and (4) has a solution.

Proof. By using (27), for $g(x) \in J\left(A_{q}^{*}\right)$, we get:

$$
\left|\phi\left(z, g(z), D_{q^{-1}} g(z), D_{q^{-1}}^{2} g(z), \ldots, D_{q^{-1}}^{j} g(z)\right)\right| \leq N,
$$

where $N:=L+\sum_{k=0}^{j} L_{k}\left(2 Q_{k}\right)^{\alpha_{k}}$. Hence, the result follows by observing that the hypothesis of Theorem 4 is satisfied and replacing $M$ by $N$ such that $Q_{k}(0 \leq k \leq j)$ are sufficiently large.

Theorem 5. Suppose that the function $\phi\left(z, g_{0}, g_{1}, \ldots, g_{j}\right)$ on the compact set $A_{q}^{*} \times E_{1}$ satisfies the following conditions:

$$
\left|\phi\left(z, g_{0}, g_{1}, \ldots, g_{j}\right)\right| \leq L+\sum_{k=0}^{j} L_{k}\left|g_{k}\right|
$$

where

$$
\begin{gathered}
E_{1}=\left\{\left(g_{0}, g_{1}, \ldots, g_{j}\right),\left|g_{k}\right| \leq \tilde{p}_{k}+C_{2 n+1, k} \frac{L+c}{1-\theta}, 0 \leq k \leq j\right\}, \\
\max _{z \in A_{q}^{*}}\left|D_{q^{-1}}^{(k)} P_{n}(z ; q)\right|=\tilde{p_{k}}, \quad c=\sum_{k=0}^{j} L_{k} \tilde{p}_{k}, \quad \theta=\sum_{k=0}^{j} C_{2 n+1, k} L_{k}<1 .
\end{gathered}
$$

Then, the boundary value problem (3) and (4) has a solution in $E_{1}$. 
Proof. Let $y(z)=g(z)-P_{n}(z ; q)$. Then, the boundary value problem (3) and (4) is equivalent to the following problem:

$$
\begin{gathered}
(-1)^{n} D_{q^{-1}}^{2 n+1} y(z)=\phi\left(z,\left(y+P_{n}\right)(z), D_{q^{-1}}\left(y+P_{n}\right), \ldots, D_{q^{-1}}^{j}\left(y+P_{n}\right)\right) \\
y(0)=\left(D_{q^{-1}}^{2 k-1} y\right)(0)=\left(D_{q^{-1}}^{2 k-1} y\right)(1)=0, \quad k=0,1, \ldots, n
\end{gathered}
$$

For $y \in C_{q^{-1}}^{j}\left(A_{q}^{*}\right)$, we define:

$$
\|y\|_{q}=\max \left\{\sup _{z \in A_{q}^{*}}\left|D_{q^{-1}}^{k} y(z)\right|, \quad 0 \leq k \leq j\right\}
$$

and we consider the operator $T_{1}: C_{q^{-1}}^{j}\left(A_{q}^{*}\right) \rightarrow C_{q^{-1}}^{j}\left(A_{q}^{*}\right)$, which is defined by:

$$
\begin{gathered}
\left(T_{1} y\right)(z)=\int_{0}^{1}\left|H_{n}(z, q s)\right| \times \\
\phi\left(s, y(s)+P_{n}(s), D_{q^{-1}}\left(y+P_{n}\right)(s), \ldots, D_{q^{-1}}^{k}\left(y+P_{n}\right)(s)\right) d_{q} s .
\end{gathered}
$$

We will use the same technique of the proof in Theorem 4 . Therefore, it is sufficient to prove that $T_{1}$ maps the set:

$$
J_{1}\left(A_{q}^{*}\right):=\left\{y(z) \in C_{q^{-1}}^{j}\left(A_{q}^{*}\right):\|y\|_{q} \leq C_{2 n+1, j} \frac{L+c}{1-\theta}\right\}
$$

into itself. For this, let $y(z) \in J\left(A_{q}^{*}\right)$. It immediately follows that:

$$
\left(\left(y+P_{n}\right)(z), D_{q^{-1}}\left(y+P_{n}\right)(z), \ldots, D_{q^{-1}}^{j}\left(y+P_{n}\right)(z)\right) \in E_{1},
$$

and then:

$$
\left|D_{q^{-1}}^{k}\left(y+P_{n}\right)(z)\right| \leq \tilde{p}_{k}+C_{2 n+1, k} \frac{L+c}{1-\theta}, 0 \leq k \leq j .
$$

Thus, from (28), (30), (31), and Lemma 5, we get:

$$
\begin{aligned}
\left|D_{q^{-1}}^{j}\left(T_{1} y\right)(z)\right| & \leq \int_{0}^{1}\left|D_{q^{-1}}^{j} H_{n}(z, q s)\right|\left(L+\sum_{k=0}^{j} L_{k}\left|D_{q^{-1}}^{k}\left(y+P_{n}\right)(s)\right|\right) d_{q s} \\
& \leq C_{2 n+1, j}\left[L+\sum_{k=0}^{j} L_{k} C_{2 n+1, k} \frac{L+c}{1-\theta}+\sum_{k=0}^{j} L_{k} \tilde{p}_{k}\right] \\
& =C_{2 n+1, j}\left[L+\theta \frac{L+c}{1-\theta}+c\right] \\
& =C_{2 n+1, j}\left[\theta \frac{L+c}{1-\theta}+\frac{(L+c)}{1-\theta}(1-\theta)\right] \\
& =C_{2 n+1, j} \frac{(L+c)}{1-\theta} .
\end{aligned}
$$

Theorem 6. Suppose that the function $\phi\left(z, g_{0}, g_{1}, \ldots, g_{j}\right)$ on the compact set $A_{q}^{*} \times E_{2}$ satisfies the Lipschitz condition:

$$
\left|\phi\left(z, g_{0}, g_{1}, \ldots, g_{j}\right)-\phi\left(z, f_{0}, f_{1}, \ldots, f_{j}\right)\right| \leq \sum_{k=0}^{j} L_{k}\left|g_{k}-f_{k}\right|,
$$


where $E_{2}$ is the same as $E_{1}$ in (29), with:

$$
L=\max _{z \in A_{q}^{*}}|\phi(z, 0,0, \ldots, 0)| .
$$

Then, the boundary value problem (3) and (4) has a unique solution in $E_{2}$.

Proof. Since the Lipschitz condition (32) implies (28), the existence of a solution follows from Theorem 5. To prove the uniqueness, let $g(z)$ and $y(z)$ be two solutions of the boundary value problem (3) and (4) in $E_{2}$. Then, as in Theorem 5, it follows that $\|g-y\| \leq \theta\|g-y\|$, and since $\theta<1$, we get $g(z)=y(z)$.

Remark 3. If $\phi=0$, then the complementary $q$-Lidstone boundary value problem (3) and (4) has a unique solution $g(z)=P_{n}(z ; q)$.

We illustrate Theorem 4 by the following example:

Example 1. Consider the complementary q-Lidstone boundary value problem:

$$
\begin{gathered}
(-1)^{n} D_{q^{-1}}^{3} g(z)=\phi\left(z, g, D_{q^{-1}} g, \ldots, D_{q^{-1}}^{j} g\right), z \in A_{q^{\prime}}^{*} \\
g(0)=q^{3}, \quad D_{q^{-1}} g(0)=-q^{3} \quad D_{q^{-1}} g(1)=q^{3},
\end{gathered}
$$

where $0 \leq j \leq 2$ is fixed and $n=1$. By using Equation (14), we can compute the $q^{-1}$-derivative of $P_{n}(z ; q)$ to get:

$$
\left(D_{q^{-1}} P_{n}\right)(z ; q)=q^{2}(2 z-1)
$$

Therefore,

$$
\begin{aligned}
& P_{n}(z ; q)=\int_{0}^{q z}\left(D_{q^{-1}} P_{n}\right)(t ; q) d_{q} t+g(0) \\
& =\frac{2 q^{4}}{1+q} z^{2}-q^{3} z+q^{3} \\
& \tilde{p_{0}}=\max _{z \in A_{q}^{*}}\left|P_{n}(z)\right|=P_{n}(0)=q^{2}, \quad \tilde{p_{1}}=\max _{z \in A_{q}^{*}}\left|D_{q^{-1}} P_{n}(z)\right|=q^{2} \text {, } \\
& \tilde{p_{2}}=\max _{z \in A_{q}^{*}}\left|D_{q^{-1}}^{(2)} P_{n}(z)\right|=2 q^{2} .
\end{aligned}
$$

We consider two cases:

Case 1. Suppose $j=0$ and $\phi(z, g)=\frac{\sqrt{q}}{12(1-q)^{2}} z g^{2}$. Then, the $q$-differential equation:

$$
(-1)^{n} D_{q^{-1}}^{(3)} g(z)=\frac{\sqrt{q}}{12(1-q)^{2}} z g^{2}, \quad z \in A_{q}^{*}
$$

with the boundary condition (34) has a solution in the set $E=\left\{g(z),|g| \leq 2 Q_{0}\right\}$, provided $M C_{3,0} \leq Q_{0}$ and $Q_{0} \geq \tilde{p_{0}}=q^{2}$ where:

$$
M=\max _{(z, g) \in A_{q}^{*} \times E}|\phi(z, g)|=\frac{\sqrt{q}}{3(1-q)^{2}} Q_{0}^{2}, \quad C_{3,0}=q^{\frac{3}{2}}(1-q)^{2},
$$

and then, $q^{2} \leq Q_{0} \leq \frac{3}{q^{2}}$.

Case 2. Suppose that $j=2$ and:

$$
\phi\left(z, g, D_{q^{-1}} g, D_{q^{-1}}^{2} g\right)=z^{2} g+\sqrt{z} D_{q^{-1}} g-D_{q^{-1}}^{2} g,
$$


and define the set:

$$
E=\left\{\left(g, D_{q^{-1}} g, D_{q^{-1}}^{2} g\right): Q_{j} \leq\left|D_{q^{-1}}^{j} g\right| \leq 2 Q_{j}, 0 \leq j \leq 2\right\} .
$$

Assume that $Q_{j} \geq \tilde{p}_{j}$ and $M C_{3, j} \leq Q_{j}, j=0,1,2$, where:

$$
M=\max _{(z, g) \in A_{q}^{*} \times E}|\phi(z, g(z))|=2 Q_{0}+2 Q_{1}-Q_{2} .
$$

By using Lemma 5 , we obtain $C_{3,0}=q^{\frac{3}{2}}(1-q)^{2}, C_{3,1}=q^{\frac{1}{2}}(1-q)^{2}$, and $C_{3,2}=q+\frac{q^{2}}{1+q}$. Thus, the condition $M C_{3, j} \leq Q_{j}$ implies that:

$$
\begin{aligned}
& {\left[\frac{1}{q^{\frac{3}{2}}(1-q)^{2}}-2\right] Q_{0}-2 Q_{1}+Q_{2} \geq 0} \\
& {\left[\frac{1}{q^{\frac{1}{2}}(1-q)^{2}}-2\right] Q_{1}-2 Q_{0}+Q_{2} \geq 0} \\
& {\left[\frac{1+q}{q+2 q^{2}}+1\right] Q_{2}-2 Q_{0}-Q_{1} \geq 0 .}
\end{aligned}
$$

Pick $Q_{0}=Q_{1}=q^{2}$ and $Q_{2}=4 q^{2}$, which satisfy (36), and the condition $Q_{j} \geq \tilde{p}_{j}$ for $j=0,1,2$. Thus, by Theorem 4, the q-differential equation:

$$
(-1)^{n} D_{q^{-1}}^{(3)} g(z)=\phi\left(z, g, D_{q^{-1}} g, D_{q^{-1}}^{(2)} g\right), \quad z \in A_{q}^{*}
$$

has a solution in $E$.

The following example illustrates Theorem 5:

Example 2. Consider the complementary q-Lidstone boundary value problem:

$$
\begin{gathered}
(-1)^{n} D_{q^{-1}}^{(3)} g(z)=z+\frac{(1-q) z}{4} g(z)+\left(\frac{z}{2}\right)^{2} D_{q^{-1}} g(z)+ \\
\frac{(q-1)^{2}}{8} D_{q^{-1}}^{(2)} g(z), \quad z \in A_{q^{\prime}}^{*}
\end{gathered}
$$

with the boundary condition (34). The interpolating polynomial $P_{q}(z)$ is given in Example 1. Note that:

$$
\left|\phi\left(z, g_{0}, g_{1}, g_{2}\right)\right| \leq 1+\frac{1}{4}|g(z)|+\frac{1}{4}\left|D_{q^{-1}} g(z)\right|+\frac{1}{8}\left|D_{q^{-1}}^{(2)} g(z)\right|, z \in A_{q}^{*} .
$$

Then, the conditions in Theorem 5 are satisfied with:

$$
\begin{gathered}
L=1, \quad L_{0}=\frac{1}{4}, \quad L_{1}=\frac{1}{4}, \quad L_{2}=\frac{1}{8} \\
c=\sum_{k=0}^{2} L_{k} \tilde{p}_{k}=\frac{3}{4} q^{2}, \quad \theta=\sum_{k=0}^{2} C_{3, k} L_{k}<\frac{3}{4}<1 .
\end{gathered}
$$


Thus, the complementary q-Lidstone boundary value problems (34)-(37) have a solution in:

$$
\begin{gathered}
E_{1}=\left\{\left(g_{0}, g_{1}, g_{2}\right), \quad\left|g_{0}\right|=|g(z)| \leq q^{2}+q^{\frac{3}{4}}(1-q)^{2} \frac{1+\frac{3}{4} q^{2}}{1-\theta}\right. \\
\left|g_{1}\right| \leq q^{2}+q^{\frac{1}{2}}(1-q)^{2} \frac{1+\frac{3}{4} q^{2}}{1-\theta}, \quad\left|g_{2}\right| \leq\left(q+\frac{q^{2}}{1+q}\right) \frac{1+\frac{3}{4} q^{2}}{1-\theta}, \\
\left.\theta=\frac{1}{4} q^{\frac{3}{4}}(1-q)^{2}+\frac{1}{4} q^{\frac{1}{2}}(1-q)^{2}+\frac{1}{8}\left(q+\frac{q^{2}}{1+q}\right)\right\} .
\end{gathered}
$$

\section{Concluding Remarks}

The $q$-Lidstone polynomials are defined in analogy with the well known Lidstone polynomials through the $q$-translation operator and the $q$-analogue of the Bernoulli polynomials. These polynomials of degree $2 n+1$ satisfy analogue conditions of the Lidstone polynomials with respect to the $q$-differential operator $D_{q}^{-1}$. It was recently proven, that under certain conditions, an entire function $f$ can be expanded with respect to the points 0 and 1 in terms of the $q$-Lidstone polynomials.

In [6], we studied the boundary value problems, which consist of an even order $q$-differential equation and the $q$-Lidstone boundary conditions. This paper extended this technique to solve some problems. We introduced the complementary $q$-Lidstone interpolating polynomial of degree $2 n$, which involves interpolating data at the odd-order $q$-derivatives in zero and one, and provided a $q$-Peano representation of the error function.

This work provided the basis for several applications that we can search in the future. Firstly, we are interested in studying the possibility of extending $q$-Lidstone and complementary $q$-Lidstone interpolation polynomials to triangular domains. The analogous problem for the classical case was posed by Agarwal and Wong [12] and studied in [13,14]. Secondly, we are interested in applying such expansions to the construction of the boundary-type quadrature formula on triangles (see [15]) or to a solution of Hermite-Birkhoff interpolation problems on scattered data (see $[16,17])$.

Author Contributions: Z.M. and M.A.-T. contributed to the design and implementation of the research, to the analysis of the results and to the writing of the manuscript. All authors have read and agreed to the published version of the manuscript.

Funding: This research received no external funding.

Acknowledgments: The authors would like to express appreciation to the editor, the referees for their helpful comments and suggestions that improved this article.

Conflicts of Interest: The authors declare no conflict of interest.

\section{References}

1. Lidstone, G. Notes on the extension of Aitken's theorem (for polynomial interpolation) to the Everett types. Proc. Edinb. Math. Soc. 1929, 2, 16-19. [CrossRef]

2. Ismail, M.; Mansour, Z. $q$-analogs of Lidstone expansion theorem, two point Taylor expansion theorem, and Bernoulli polynomials. Anal. Appl. 2018. [CrossRef]

3. Gasper, G.; Rahman, M. Basic Hypergeometric Series; Cambridge University Press: Cambrdge, UK, 2004.

4. Jackson, F. On $q$-functions and a certain difference operator. Earth Environ. Sci. Trans. R. Soc. Edinb. 1909, 46, 253-281. [CrossRef]

5. Annaby, M.H.; Mansour, Z. q-Fractional Calculus and Equations; Springer: Berlin, Germany, 2012.

6. Mansour, Z.; Al-Towailb, M. $q$-Lidstone polynomials and existence results for $q$-boundary value problems. Bound. Value Probl. 2017. [CrossRef]

7. Costabile, F.; Dell'Accio, F.; Luceri, R. Explicit polynomial expansions of regular real functions by means of even order Bernoulli polynomials and boundary values. J. Comput. Appl. Math. 2005, 175, 77-99. [CrossRef]

8. Agarwal, R.P.; Pinelas, S.; Wong, P.J.Y. Complementary Lidstone interpolation and boundary value problems. J. Inequal. Appl. 2009. [CrossRef]

9. Schultz, M. Error bounds for polynomial spline interpolation. Math. Comput. 1970, 24, 507-515. [CrossRef] 
10. Budakçi, G.; Oruç, H. A generalization of the Peano kernel and its applications. Fac. Sci. Univ. Ank. Ser. A1 Math. Stat. 2018, 67, 229-241.

11. Arzelà, C. Sulle funzioni di linee. Mem. Accad. Sci. Ist. Bologna Cl. Sci. Fis. Mat. 1895, 5, 55-74.

12. Agarwal, R.P.; Wong, P.J.Y. Error Bounds for the Derivatives of Lidstone Interpolation and Applications. Pure Appl. Math. 1998, 212, 1-41.

13. Costabile, F.A.; Dell'Accio, F. Lidstone approximation on the triangle. Appl. Numer. Math. 2005, 52, 339-361. [CrossRef]

14. Costabile, F.A.; Dell'Accio, F.; Guzzardi, L. New bivariate polynomial expansion with boundary data on the simplex. Calcolo 2008, 45, 177-192. [CrossRef]

15. Costabile, F.A.; Dell'Accio, F. New embedded boundary-type quadrature formulas for the simplex. Numer. Algorithms 2007, 45, 253-267. [CrossRef]

16. Caira, R.; Dell'Accio, F.; Di Tommaso, F. On the bivariate Shepard-Lidstone operators. J. Comput. Appl. Math. 2012, 236, 1691-1707. [CrossRef]

17. Costabile, F.A.; Dell'Accio, F.; Di Tommaso, F. Complementary Lidstone interpolation on scattered data sets. Numer. Algorithms 2013, 64, 157-180. [CrossRef]

(C) 2020 by the authors. Licensee MDPI, Basel, Switzerland. This article is an open access article distributed under the terms and conditions of the Creative Commons Attribution (CC BY) license (http://creativecommons.org/licenses/by/4.0/). 